\title{
Complicated Secondary Pneumonia after Swine-Origin Influenza A Virus Infection in an Immunocompetent Patient
}

\author{
Ryotaro Igusa, ${ }^{1}$ Tomohiro Sakakibara, ${ }^{2}$ Taizo Shibahara, ${ }^{2}$ Kazuhiro Sakamoto, ${ }^{3}$ \\ Hidekazu Nishimura ${ }^{4}$ and Kozo Ota ${ }^{5}$ \\ ${ }^{1}$ Department of Respiratory Medicine, Osaki Citizen Hospital, Osaki, Japan \\ ${ }^{2}$ Department of Respiratory Medicine, Tohuku University Hospital, Sendai, Japan \\ ${ }^{3}$ Department of Pathology, Osaki Citizen Hospital, Osaki, Japan \\ ${ }^{4}$ Department of Virus Research Center, Clinical Research Division, Sendai Medical Center, Sendai, \\ Japan \\ ${ }^{5}$ Department of Medicine, Osaki Citizen Hospital, Osaki, Japan
}

The pandemic of the swine-origin influenza A virus (S-OIV) in 2009 demonstrated severe viral pneumonia followed by acute respiratory distress syndrome (ARDS). Although ARDS would be caused by the influenza virus pneumonia itself, it has remained unclear whether other respiratory viral or bacterial infections coexist with S-OIV pneumonia. We report an immunocompetent patient with methicillin-resistant Staphylococcus aureus (MRSA) and Herpes simplex virus (HSV) pneumonia secondary to S-OIV infection. A 57-year-old man previously without major medical illness was admitted to our hospital with severe pneumonia accompanied by ARDS due to S-OIV. In his clinical course, anti-influenza treatment was not effective. Sputum culture revealed the presence of MRSA, and HSV was isolated in broncho-alveoler lavage (BAL) fluid. Administration of an antiviral agent (acyclovir), an antibacterial agent (linezolid), and a corticosteroid (methylprednisolone) successfully improved the pneumonia and ARDS. HSV pneumonia can scarcely be seen in healthy people. However recently it has been recognized as a ventilator-associated pneumonia. Although coexistence of Streptococcus pneumoniae and MRSA was reported in S-OIV pneumonia, secondary viral infection has not been reported. The present report is the first patient with HSV pneumonia secondary to S-OIV infection. We propose that a possibility of hidden HSV pneumonia should be taken into consideration in patients with prolonged severe pneumonia due to influenza infection.

Keywords: acute respiratory distress syndrome; herpes simplex virus pneumonia; methicillin-resistant Staphylococcus aureus; swine-origin influenza virus; ventilator-associated pneumonia

Tohoku J. Exp. Med., 2012, 226 (2), 117-120. (C) 2012 Tohoku University Medical Press

The pandemic of the swine-origin influenza A virus (S-OIV) in 2009 resulted in a high incidence of severe influenza viral pneumonia and acute respiratory distress syndrome (ARDS), especially in children and young healthy adults (Perez-Padilla et al. 2009). Although the mortality rate for 2009 S-OIV infection varied among countries, S-OIV pneumonia was the leading cause of death in $\mathrm{S}-\mathrm{OIV}$ infection. The characteristics of viral pneumonia are bilateral diffuse infiltration by chest X-ray, severe hypoxia (sometimes requiring mechanical ventilation), rapid deterioration of respiration, and high mortality rate (Perez-Padilla et al. 2009; Bautista et al. 2010). These clinical features also indicate other concomitant viral or bacterial infections in such critically ill patients, but bacterial pneumonia has been suspected or diagnosed only in approximately $20 \%$ of patients hospitalized in intensive care units (ICUs) (Bautista et al. 2010). Therefore, it remains unclear whether second- ary viral or bacterial infection coexists with and contributes to the severity of S-OIV infection.

Herpes simplex virus (HSV) pneumonia is a rare in common populations, but is often seen in immunocompromised hosts such as those who receive corticosteroid therapy, have malignant diseases, or have undergone bone marrow or organ transplantations. HSV pneumonia has also been recognized as a component of ventilator-associated pneumonia (VAP) during prolonged mechanical ventilation of immunocompetent patients (Luyt et al. 2007). HSV pneumonia has been thought to result from systemic inflammation, mechanical ventilation stress, and respiratory distress, any of which can cause HSV reactivation from latent infection of the upper respiratory tract; the lower respiratory tract and alveolar tissues are then infected with activated HSV (Luyt et al. 2007).

The direct methods to know HSV infection are detec-

Received October 11, 2011; revision accepted for publication January 6, 2012. doi: 10.1620/tjem.226.117

Corresponding: Ryotaro Igusa, M.D., Ph.D., Department of Respiratory Medicine, Osaki Citizen Hospital, Furukawa Senjyujimati 2-3-

10, Osaki, Miyagi 989-6183, Japan.

e-mail: ryoigusa@h-osaki.jp 
tion of HSV DNA by polymerase chain reaction (PCR) and/ or isolation of HSV. However, the definition of active HSV pneumonia is still controversial. A recent report suggests that the detection of characteristic HSV infected cells by cytological examination from broncho-alveoler lavage (BAL) fluid could be the useful method to detect HSV pneumonia (Cunha et al. 2010).

We report an immunocompetent patient with methicillin-resistant Staphylococcus aureus (MRSA) and HSV pneumonia secondary to S-OIV infection.

\section{Clinical Findings of the Patient}

A 57-year-old man who had a history of depression treated with anti-depressants, presented with fever and chills, followed by severe cough and short breath. At the time of clinic visit, the patient was already in hypoxia; chest $\mathrm{X}$-ray showed bilateral diffuse infiltration. The patient was referred to our hospital and immediately transferred to the ICU due to acute respiratory distress (ARDS). The individual's medical history was noncontributory; the patient denied tobacco smoking and alcohol use and had no allergies. Medication on admission was anti-depressants only, and the patient had never taken any corticosteroid or immunosuppressant. Physical findings were as follows: body temperature, $39.0^{\circ} \mathrm{C}$; blood pressure, $150 / 99 \mathrm{mmHg}$; heart rate, 94/min; respiratory rate, $24 / \mathrm{min}$; oxygen saturation, $88 \%$ on $100 \%$ oxygen; clear consciousness; pale conjunctiva, not injected; no palpable neck lymph node; equal breath sound; fine crackles in all lung zones on both sides; normal S1 and S2 heart sound, no murmurs; no abdominal distention; normal bowel sound; no edema or clubbing; and no rush or vesiculation of the skin. The leukocyte count was $4,660 / \mathrm{mm}^{3}$ with normal differential count and erythrocytic cell indexes, and the platelet count was $149,000 / \mathrm{mm}^{3}$. The blood chemistry data were also within normal limits, except for the lactate dehydrogenase level of $807 \mathrm{IU} / \mathrm{L}$, creatinine phosphokinase level of 1,223 IU/L, aspartate aminotransferase level of $90 \mathrm{IU} / \mathrm{L}$, and C-reactive protein level of $21.8 \mathrm{mg} / \mathrm{L}$. The coagulation profile was within normal limits. Chest X-ray and computed tomography (CT) scan of the chest showed bilateral diffuse consolidation that was worse in the right lung (Fig. 1A). Electrocardiogram and echocardiogram were within normal limits. Other pertinent negative laboratory studies were blood culture, sputum culture including acid fast bacilli, human immunodeficiency virus antibody, and urine Legionella pneumophila and Streptococcus pneumoniae antigens. A rapid influenza virus antigen detection test was positive for influenza type A virus; and it was subsequently revealed to be S-OIV virus by polymerase chain reaction (PCR) test performed at laboratory of Miyagi Prefecture Public Health Institute.

The patient was placed on a mechanical ventilator for ARDS due to S-OIV pneumonia, and oseltamivir was administered. On the 6th day after hospitalization, sputum culture from the endotracheal aspirate revealed the presence of MRSA. Vancomycin was additionally administered intravenously for the possible VAP (Fig. 2). However, subsequent chest X-ray did not show any improvement despite the administration of oseltamivir and vancomycin (Fig. 1B). The antibacterial medication was changed from vancomycin to linezolid. Bronchoscopic examination was performed in order to explore the unexplained failure to wean. Culturing of BAL fluid was negative, but cytological examination revealed multinucleated giant cells that had nuclear
A

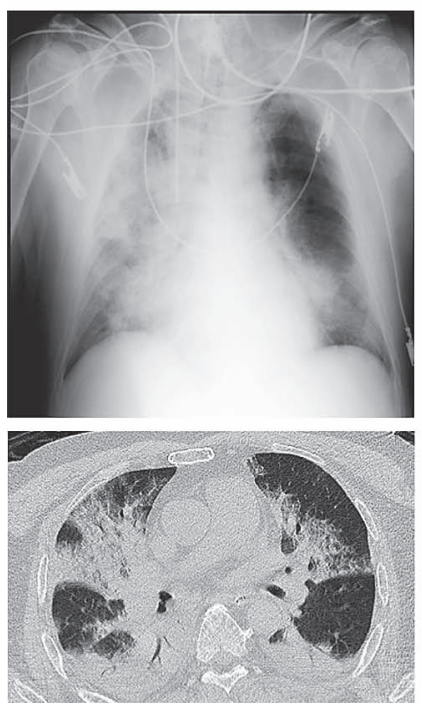

B
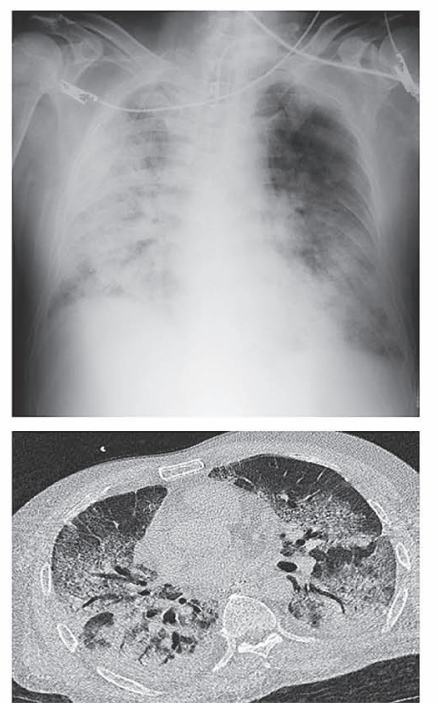
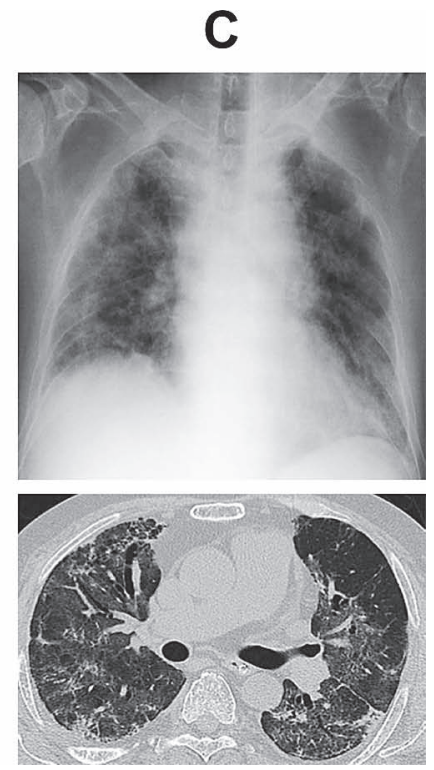

Fig. 1. Chest X-ray and CT scan of the chest.

Chest X-rays (upper panels) and CT scans of the chest (lower panels) on admission (Fig. 1A), before the first bronchoscopic examination (Fig. 1B), and at the time of discharge (Fig. 1C). In both Fig. 1A and 1B, bilateral diffuse infiltration (chest X-rays) and air-space consolidation (CT scans) can be seen. Acyclovir and methylprednisolone ameliorated the infiltration and air space consolidation (Fig. 1C). 


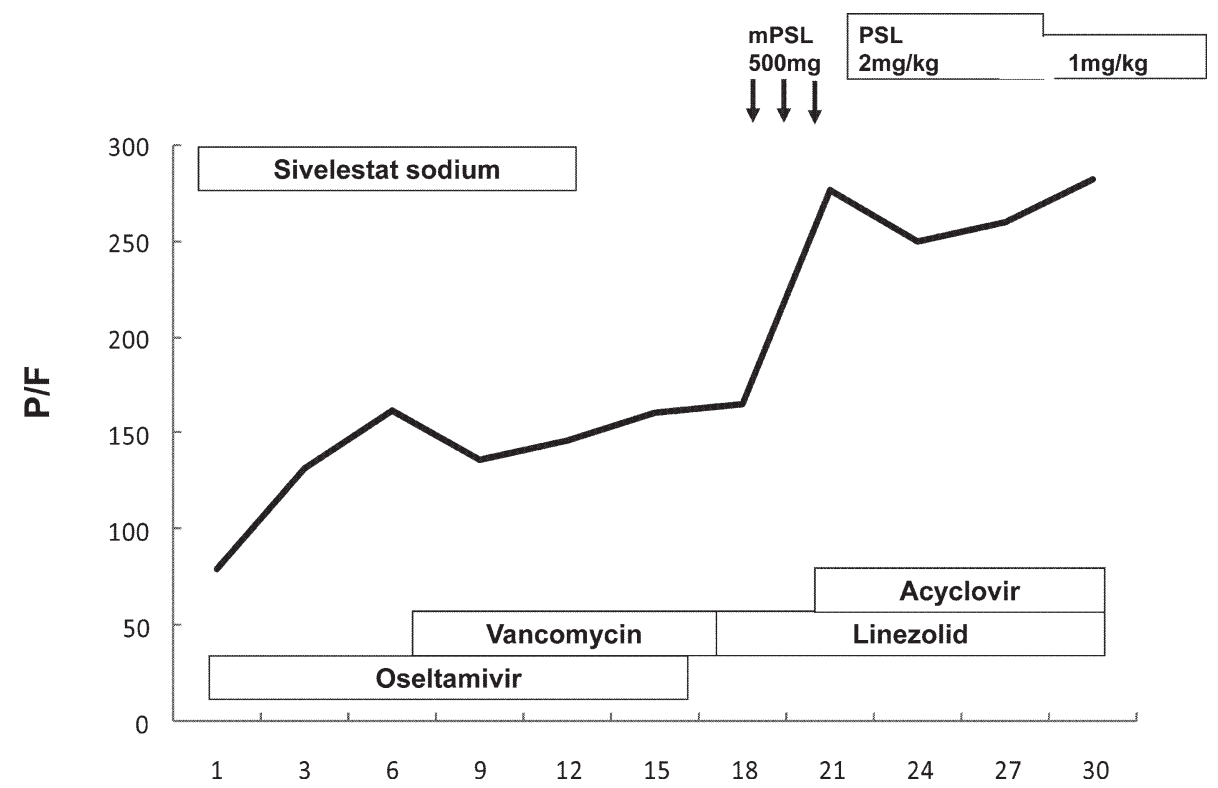

Fig. 2. Clinical course of the case.

The ratio of $\mathrm{PaO} 2$ to $\mathrm{FiO} 2$ (denoted as $\mathrm{P} / \mathrm{F}$; $\mathrm{y}$-axis) is plotted as a function of the number of days after admission (x-axis).

Following treatment with methylprednisolone (mPSL), predonisolone (PSL), linezolid, and acyclovir, the P/F ratio was clearly improved.

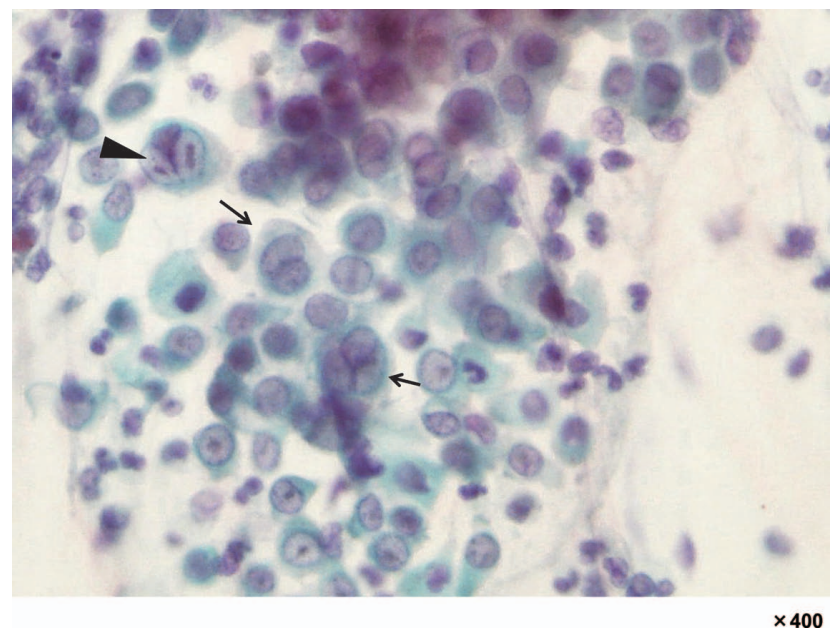

Fig. 3. Papanicolaou stain of BAL fluid cells. Arrows indicate multinucleated giant cells. Arrow head indicates ground-glass appearance nucleus with intranuclear inclusion body. Magnification, $\times 400$.

ground-glass appearance and inclusion bodies (Fig. 3). These characteristic cytological findings suggested active HSV-infected cells. Viral culture at the Virus Research Center, Sendai Medical Center revealed HSV infection. Therefore, acyclovir and methylprednisolone were administered for probable HSV pneumonia and late-phase ARDS, respectively (Fig. 2). These treatments alleviated the chest findings as judged by chest X-ray and CT scan, and improved the oxygenation status (Fig. 1C). The patient was extubated on the following 10th day after the start of administration of acyclovir and methylprednisolone.

At the time of the second bronchoscopic examination
(27 days after the first examination), cytological examination did not show any characteristic HSV-infected cells. Viral culture on the same sample did not show HSV infection. An immunoglobulin $\mathrm{G}$ ( $\operatorname{IgG}$ ) antibody test performed just prior to the initiation of acyclovir therapy indicated past HSV infection; 6 weeks later, the serum anti-HSV IgG level had doubled as compared to the value before treatment.

\section{Discussion}

S-OIV pandemic of 2009 demonstrated clinical aspects characteristic of the virus. S-OIV is reported as a rapidly progressive pneumonia with ARDS among children and young adult patients, who initially presented typical influenza-like illness followed by dyspnea and tachypnea and intubation was often necessary within 24 hours after admission (Perez-Padilla et al. 2009; Bautista et al. 2010). This rapid deterioration of clinical course is caused primarily by S-OIV infection and its rapid replication in the respiratory epithelium. One possible mechanism of respiratory failure was suggested that S-OIV might directly injure the alveolar epithelial cells, and furthermore, subsequently occurred cytokine storm might cause diffuse alveolar damage (Bautista et al. 2010), which is characteristic of ARDS and systemic inflammatory response syndrome. This patient presented with typical influenza symptoms and rapidly developed respiratory failure; a CT scan of the chest showed bilateral diffuse infiltration. These clinical features corresponded to those previously reported for S-OIV pneumonia with ARDS (Perez-Padilla et al. 2009; Bautista et al. 2010).

Bacterial pneumonia has only been diagnosed in about $20 \%$ of patients in the IUC, and has been found in $26 \%$ to 
$38 \%$ of patients who died from S-OIV infection (Bautista et al. 2010). S-OIV pneumonia often shows a short clinical course from admission to decease when bacterial pneumonia coexists (Bautista et al. 2010). Although oseltamivir was administered, the patient's chest X-ray did not show any improvement; therefore concomitant bacterial pneumonia was suspected. Vancomycin and linezolid were additionally administered for the possible VAP according to the result of the culture from endotracheal aspirates. However, this antibiotic therapy did not improve the patient's pneumonia with ARDS. This individual's ongoing condition suggested that oseltamivir might have been effective in treating the S-OIV infection itself, but that ARDS had persisted due to other unexpected complications, as evidenced by a secondary viral infection in addition to bacterial pneumonia.

HSV pneumonia recently has been recognized as lateonset VAP in critically ill immunocompetent patients in the ICU (Luyt et al. 2007). HSV is commonly present in the oropharynx as a latent infection in immunocompetent hosts, but in critically ill patients, HSV can be reactivated by the stress of acute illness and the mechanical stress of prolonged mechanical ventilation (Luyt et al. 2007). The risk factors for HSV pneumonia are oral-labial lesions, positive throat swab cultures, and macroscopic bronchial lesions, and HSV pneumonia is observed in approximately $20 \%$ of patients who have been mechanically ventilated for 5 days or more in the ICU (Luyt et al. 2007). Patients with HSV pneumonia tend to have poor prognoses, longer durations of mechanical ventilation and ICU stays, and acquire bacterial VAP (Luyt et al. 2007). A clinical clue to the presence of HSV pneumonia among patients under mechanical ventilation is the unexplained failure to wean from ventilator despite appropriate antibiotic administration. Although typical herpetic erosive and ulcerative lesions were not found by physical and bronchoscopic examination of the upper and lower respiratory tracts, this patient had been on prolonged mechanical ventilation despite oseltamivir and antibiotic administration for S-OIV infection and possible VAP, respectively. In the context of his clinical course, this case was susceptible to HSV reactivation and infection.

The definition of HSV pneumonia remains obscure. Detection of HSV by cell culture from BAL fluid is a useful method (Numazaki et al. 1987). Cytological examination can directly reveal HSV-infected cells, which are characteristically multinucleated giant cells that have eosinophilic intranuclear inclusions and ground-glass appearance of the nucleus (Cunha et al. 2010). Cytological examination of BAL fluid in our case revealed cells with just such features. These characteristic cells were not found in BAL fluid obtained during a second bronchoscopic examination performed after acyclovir treatment. Taken together, these results indicate that this patient had HSV pneumonia that was successfully treated with acyclovir.

The use of corticosteroids in ARDS remains controversial. In a randomized, placebo-controlled study, methylprednisolone did not show a survival benefit, but did result in increased ventilator-free days and improved oxygenation (Steinberg et al. 2006). Moreover, a recent meta-analysis concluded that the use of low-dose corticosteroids was associated with improved mortality and morbidity (Meduri et al. 2009). These reports suggest that corticosteroids might be beneficial for selected patients with ARDS. In this patient, acyclovir was effective for treatment of HSV pneumonia and early-phase ARDS, but methylprednisolone is believed to have contributed to efficacy against late-phase ARDS.

In conclusion, this study reports HSV pneumonia secondary to S-OIV pneumonia with ARDS. Acyclovir and methylprednisolone therapy successfully cured the patient. We propose that HSV pneumonia should be considered as a complication of S-OIV pneumonia with ARDS.

\section{Conflict of Interest}

The authors have no conflict of interest to declare in relation to this article.

\section{References}

Bautista, E., Chotpitayasunondh, T., Gao, Z., Harper, S.A., Shaw, M., Uyeki, T.M., Zaki, S.R., Hayden, F.G., Hui, D.S. \& Kettner, J.D. (2010) Clinical aspects of pandemic 2009 influenza A (H1N1) virus infection. N. Engl. J. Med., 362, 17081719.

Cunha, B.A., Chak, A. \& Durie, N. (2010) Herpes simplex virus-1 late-onset ventilator-associated pneumonia: the importance of cytopathologic diagnosis. Am. J. Infect. Control, 38, 249-250.

Luyt, C.E., Combes, A., Deback, C., Aubriot-Lorton, M.H., Nieszkowska, A., Trouillet, J.L., Capron, F., Agut, H., Gibert, C. \& Chastre, J. (2007) Herpes simplex virus lung infection in patients undergoing prolonged mechanical ventilation. Am. J. Respir. Crit. Care Med., 175, 935-942.

Meduri, G.U., Annane, D., Chrousos, G.P., Marik, P.E. \& Sinclair, S.E. (2009) Activation and regulation of systemic inflammation in ARDS: rationale for prolonged glucocorticoid therapy. Chest, 136, 1631-1643.

Numazaki, Y., Oshima, T., Ohmi, A., Tanaka, A., Oizumi, Y., Komatsu, S., Takagi, T., Karahashi, M. \& Ishida, N. (1987) A microplate method for isolation of viruses from infants and children with acute respiratory infections. Microbiol. Immunol., 31, 1085-1095.

Perez-Padilla, R., de la Rosa-Zamboni, D., Ponce de Leon, S., Hernandez, M., Quinones-Falconi, F., Bautista, E., RamirezVenegas, A., Rojas-Serrano, J., Ormsby, C.E. \& Corrales, A. (2009) Pneumonia and respiratory failure from swine-origin influenza A (H1N1) in Mexico. N. Engl. J. Med., 361, 680-689.

Steinberg, K.P., Hudson, L.D., Goodman, R.B., Hough, C.L., Lanken, P.N., Hyzy, R., Thompson, B.T. \& Ancukiewicz, M. (2006) Efficacy and safety of corticosteroids for persistent acute respiratory distress syndrome. N. Engl. J. Med., 354, 1671-1684. 